\title{
To investigate the effect of grass-based feeding practices on insulin sensitivity, adipose tissue inflammation and hepatic lipid composition in a transgenic mouse model
}

\author{
Y.M. Lenighan ${ }^{1}$, M.E. O’Reilly ${ }^{1}$, S. Kajani ${ }^{2}$, S. B. Curley ${ }^{2}$, R. Bruen ${ }^{2}$, Sabine M. Harrison ${ }^{3}$, \\ Aidan P. Maloney ${ }^{4}$, Frank J. Monahan ${ }^{3}$, F.C. McGillicuddy ${ }^{2}$ and H.M. Roche ${ }^{1}$ \\ ${ }^{1}$ Nutrigenomics Research Group, Conway Institute of Biomedical and Biomolecular Research, \\ ${ }^{2}$ Diabetes Complications Research Centre, UCD Conway Institute of Biomedical and Biomolecular Research, \\ ${ }^{3}$ Institute of Food and Health and School of Medicine, University College Dublin, Belfield, Dublin, Ireland and \\ ${ }^{4}$ Teagasc, Grange Research Centre, Dunsany, Co. Meath, Ireland.
}

Grass-based feeding practices can alter the fatty acid composition of beef to reduce saturated fatty acid (SFA) concentrations and increase polyunsaturated fatty acid (PUFA) concentrations, in particular, $\alpha$-linolenic acid (ALA) and conjugated linoleic acid $(C L A){ }^{(1)}$. The beneficial effect of chronic grass-fed red meat consumption on LC n-3 PUFA status in human has previously been characterised $^{(2)}$. However, the in vivo mechanism is currently unknown. Therefore the aim of this study was to assess the impact of beef consumption from alternate feeding practices on insulin sensitivity, adipose tissue inflammation and hepatic lipid composition. ApoE.3L.CETP transgenic mice were fed either a GRASS (18\% beef protein, $30 \%$ beef fat, derived from grass-fed beef) a CONC (18\% beef protein, $30 \%$ beef fat, derived from concentrate-fed beef) or a GRASS-CASEIN (18\% casein, $30 \%$ grass-fed beef fat) diet for 12 weeks. Following the dietary intervention, the metabolic phenotype was characterised using glucose tolerance tests (GTT) and insulin tolerance tests (ITT). Epididymal adipose tissue was harvested to isolate the stromal vascular fraction (SVF) cellular fraction which is enriched in immune cells and stimulated with 100ng/ml LPS for 18 hours, and hepatic lipid composition was characterised using a two-step microwave-assisted saponification and esterification process. Resultant adipose IL-6 secretion was measured by ELISA. Prism version 5 (GraphPad Software Inc.) was used for statistical analysis. One- and two-way ANOVA tested for differences between diets in the GTT, ITT, adipose cytokine secretion and hepatic fatty acid composition.

No difference in glucose tolerance, insulin sensitivity of adipose tissue inflammation was observed between the beef interventions. However, significant differences in hepatic fatty acid composition were observed following the dietary intervention, wherein PUFA concentrations were increased following the GRASS diets (Table 1). In conclusion, this work demonstrates the potential of grass-fed beef consumption to improve fatty acid status. Future perspectives include a chronic intervention with grass-fed beef.

Table 1. Fatty acid composition of livers following beef dietary intervention in transgenic mouse model

\begin{tabular}{|c|c|c|c|c|c|c|c|}
\hline & \multicolumn{2}{|l|}{$\begin{array}{l}\text { GRASS } \\
(n=7)\end{array}$} & \multicolumn{2}{|l|}{$\begin{array}{l}\text { CONC } \\
(n=8)\end{array}$} & \multicolumn{2}{|c|}{$\begin{array}{l}\text { GRASS-CASEIN } \\
(n=6)\end{array}$} & \multirow[b]{2}{*}{$\mathrm{P}^{*}$} \\
\hline & Mean & $S D$ & Mean & $\overline{S D}$ & Mean & $S D$ & \\
\hline C16:0 Palmitic Acid & $18.39^{\mathrm{a}}$ & 3.17 & $14.74^{\mathrm{a}}$ & 3.97 & $27.92^{\mathrm{b}}$ & 9.78 & 0.002 \\
\hline C16: 1 Palmitoleic Acid & $6.59^{\mathrm{a}}$ & 1.35 & $4.96^{\mathrm{a}}$ & 2.03 & $13.17^{\mathrm{b}}$ & 5.45 & 0.001 \\
\hline C18:0 Stearic Acid & 3.26 & 0.17 & 2.97 & 0.29 & 3.1 & 0.29 & 0.119 \\
\hline C18:1 Oleic Acid & $54.71^{\mathrm{ab}}$ & 12.82 & $38.74^{\mathrm{a}}$ & 12.87 & $75.34^{\mathrm{b}}$ & 30.88 & 0.01 \\
\hline C18:2n-6 Linoleic Acid & 8.38 & 1.3 & 6.17 & 1.98 & 6.52 & 3.77 & 0.214 \\
\hline C18:3n-3 Alpha Linolenic Acid & $0.46^{\mathrm{a}}$ & 0.09 & $0.22^{\mathrm{b}}$ & 0.1 & $0.40^{\mathrm{a}}$ & 0.16 & 0.002 \\
\hline C18:2c9t11 Conjugated Linoleic Acid & $1.32^{\mathrm{a}}$ & 0.19 & $0.31^{\mathrm{b}}$ & 0.11 & $1.50^{\mathrm{a}}$ & 0.45 & $<0.001$ \\
\hline C18:2t10c12 Conjugated Linoleic Acid & $0.04^{\mathrm{a}}$ & 0.02 & $0.01^{\mathrm{b}}$ & 0.01 & $0.06^{\mathrm{a}}$ & 0.04 & 0.004 \\
\hline C20:4n-6 Arachidonic Acid & $2.71^{\mathrm{a}}$ & 0.39 & $3.06^{\mathrm{a}}$ & 0.38 & $2.37^{\mathrm{b}}$ & 0.21 & 0.006 \\
\hline C20:5n-3 Eicosapentaenoic Acid & $0.40^{\mathrm{a}}$ & 0.07 & $0.19^{\mathrm{b}}$ & 0.04 & $0.25^{\mathrm{b}}$ & 0.07 & $<0.001$ \\
\hline C22:5n-3 Docosapentaenoic Acid & $0.50^{\mathrm{a}}$ & 0.06 & $0.26^{\mathrm{b}}$ & 0.08 & $0.28^{\mathrm{b}}$ & 0.09 & $<0.001$ \\
\hline C22:6n-3 Docosahexaenoic Acid & $3.10^{\mathrm{a}}$ & 0.5 & $2.27^{\mathrm{b}}$ & 0.34 & $2.42^{\mathrm{b}}$ & 0.27 & 0.002 \\
\hline
\end{tabular}

*A one way ANOVA was applied to test for differences between beef groups. ${ }^{\mathrm{a}, \mathrm{b}}$ Indicates significant differences between beef dietary interventions $(\mathrm{P}<0.05)$

This project was funded by the Irish Department of Agriculture, Food and the Marine, 'Healthy Beef' Programme (13/F/514).

1. Daley CA, Abbott A, Doyle PS et al. (2010) Nutrition Journal 910

2. McAfee AJ, McSorley EM, Cuskelly GJ et al. (2011) Br J Nutr 105 80-89 